\title{
Identification of the material properties of composite beams: inverse method approach
}

\author{
E. Euler ${ }^{1}$, H. Sol ${ }^{1} \&$ E. Dascotte ${ }^{2}$ \\ ${ }^{I}$ Department of Mechanics of Materials and Construction, \\ Vrije Universiteit Brussel, Brussels, Belgium \\ ${ }^{2}$ Dynamic Design Solutions NV, Leuven, Belgium
}

\begin{abstract}
In load carrying applications one is mainly interested in the stiffness properties of the structural component. Elastic and shear moduli supplemented with section properties render the stiffness properties of the component. Moduli of isotropic materials are well known and well documented. However, this is not the case for composite materials.

The developed procedure belongs to the group of mixed numerical experimental methods. The method makes use of modal data to determine the effective orthotropic material properties of composite beams. Modal reference data is experimentally obtained from the beam at hand. The other modal data set is obtained from a finite element model of the same beam. The orthotropic material properties, also called parameters, in the finite element model are then modified in such a way that both sets of modal data match. If those two sets match, the virtual model has "in a global sense" the same mass and stiffness properties as the real model.

A program written in FEMtools is applied to five test cases. Results are discussed.

Keywords: inverse methods, FEA, orthotropic material properties, composite materials, modal data.
\end{abstract}

\section{Introduction}

Most engineers have considerable experience in the design of simple structural components using isotropic materials. However, today more and more composite materials are used for structural elements. 
In load carrying applications one is mainly interested in the stiffness properties of the structural component. Elastic and shear moduli supplemented with section properties render the stiffness properties of the component. Moduli of isotropic materials are well known and well documented. This is not the case for composite materials. The effective laminate properties depend on fiber material, matrix material, ply orientation, laminate thickness, stacking sequence, etc. Infinite combinations are possible, resulting in a huge amount of different moduli. It is clear that not all possible scenarios are studied in literature.

If all the details of the composite material are known, the engineer can calculate effective laminate properties and use them in theories like for example the first-order shear deformation theory for thin-walled laminated beams. If the ply lay-up results in coupling phenomena, the engineer has to use advanced software to simulate the behaviour of the structure. All this requires substantial effort.

In some cases, not all the details of the composite material are known. In this case, elastic properties can be determined by experiment. Drawback, these experiments are destructive in nature. Another possibility is to obtain stiffness properties of the whole structure by conducting an experiment. Drawback here, influence of boundary conditions. All this requires substantial effort.

This paper presents a method to determine the global effective orthotropic material properties of composite beams by measuring a certain amount of natural frequencies of the structure.

\section{Outline: method and program}

First, experimental modal analysis (EMA) [1] is used to extract the natural frequencies of the physical structure. This modal data is used as reference response data during the procedure.

Next, a mathematical model of the structure is created. In the physical model all mass and stiffness related properties are known except for the anisotropic material properties. All known properties are implemented as such into the mathematical model. The real composite material is modelled as a global homogeneous orthotropic material in the mathematical model. This mathematical model is solved for modal data using finite element analysis.

Finally, two sets of non matching modal data are available. One set composed of experimentally obtained reference data. The other set contains calculated data from the mathematical model. The orthotropic material properties are then modified in such a way that both sets of modal data match. If those two sets match, the virtual model has "in a global sense" the same mass and stiffness properties as the real model. This principle is called model updating.

The procedure to identify orthotropic material properties by natural frequency measurement is automated in the form of a user-friendly FEMtools program. Program flow is visualized in the next flowchart. 


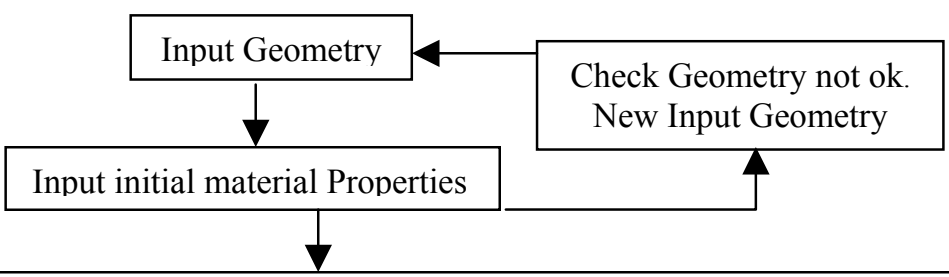

Obtain converged mesh with respect to first ten frequencies $\{\text { freq }\}_{\text {analyt }}$. The frequencies $\{\text { freq }\}_{\text {analyt }}$ are based on initial material properties. The ten $\{\text { freq }\}_{\text {analyt }}$ and $\{\psi\}_{\text {analyt }}$ are shown.

The sensitivity matrix material properties versus frequencies $\{\text { freq }\}_{\text {analyt }}$ is shown.

Determine how many $\mathrm{N}$ response frequencies $\{\text { freq }\}_{\exp }$ are taken into account.

Determine which material properties are identified.

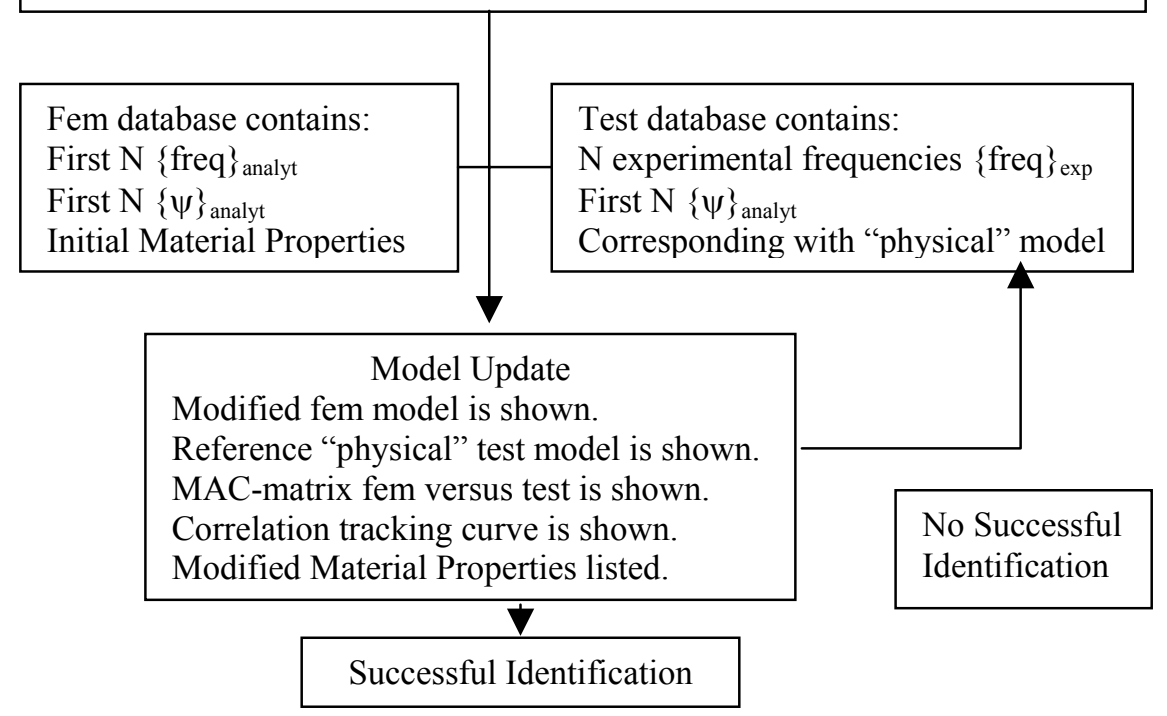

\section{Model updating: mathematics}

From a mathematical point of view, the difficulty with model updating is that the relation between output vector and the parameter vector is nearly always nonlinear. This means that updating the parameter values from an initial value to a final value has to be done iteratively. The value of the output for some new parameter values can be estimated with a Taylor expansion. The Taylor series can be cut off after the linear term or can be cut after some higher order terms. Figure 1 illustrates the mathematics involved in model updating.

The matrix $[\mathbf{S}]$ that appears in the linear Taylor term is called the sensitivity matrix. It contains the partial derivatives of the output components for the different parameter values. The success of model updating is highly dependant 
from the numerical condition of the sensitivity matrix because $[\mathbf{S}]$ must be inverted in every iteration step to obtain the parameter correction $\{\Delta \mathbf{p}\}$.
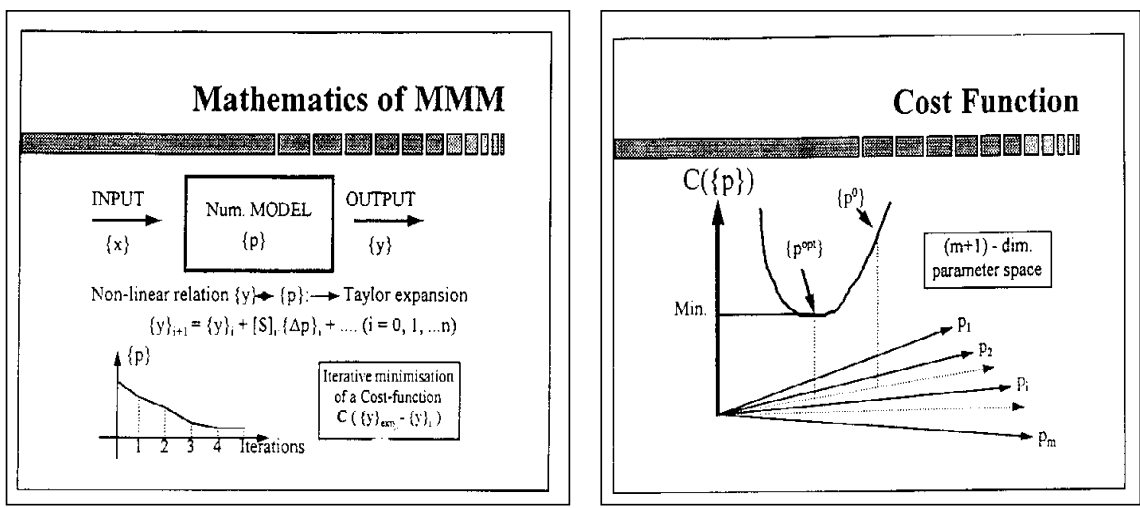

Figure 1: Mathematics of model updating (left) and minimizing of cost function (right).
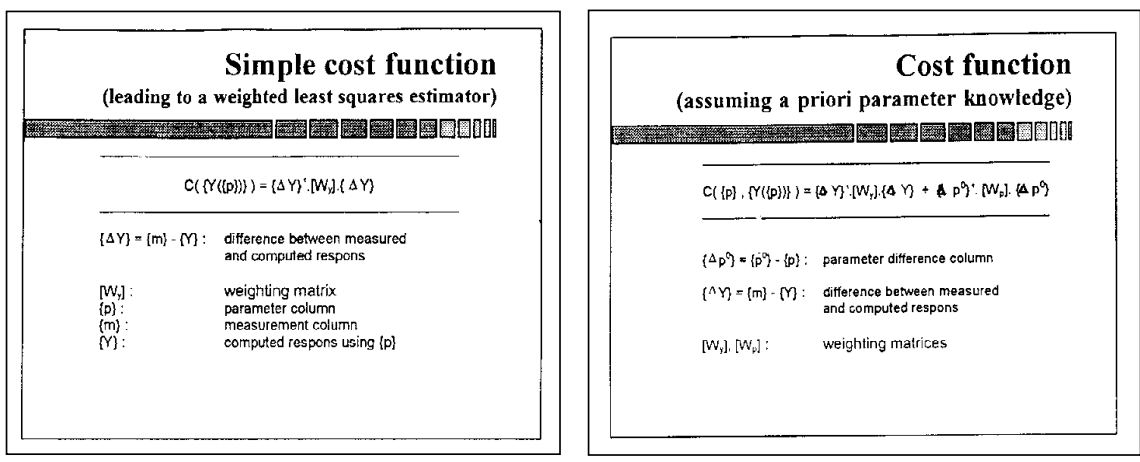

Figure 2: Simple cost function (left) and (right) a more elaborated cost function.

Convergence from an initial parameter value $\{\mathbf{p}\}_{0}$ to the final value is obtained by minimization of a cost function in every iteration step. Graphically, this means that the cost function evolutes iteratively from an initial point in the $(\mathrm{m}+1)$ dimensional parameter space towards a global minimum. The parameter values in the global minimum are the optimal parameter values. Figure 1 illustrates the principle.

One of the most simple cost functions leads to a weighted least squares estimator. In this cost function, a weighting matrix $[\mathbf{W}]$ is pre- and post multiplied with the difference between the measured and the computed response 
in a point in the parameter space. The weighting matrix $[\mathbf{W}]$ allows to express a different confidence in the different measured data points. This is illustrated in Figure 2.

A more elaborated cost function also takes the initial parameter values into account. A second term is added to the cost function in which a weighting matrix is pre- and post multiplied with the difference between the initial and the current parameter value. Again, a weighting matrix allows to express a different confidence in the different initial parameter values.

The matrix $[\mathbf{W}]_{\mathrm{p}}$ represents the weighting matrix expressing the confidence in the model parameters, while $[\mathbf{W}]_{y}$ is a weighting matrix expressing the confidence in the reference response test data.

\section{Model updating: important considerations}

The success of model updating strongly depends on the following considerations.

\subsection{Accuracy numerical model}

A first aspect is the quality of the mathematical model. All known mass and stiffness properties must be correctly represented in the mathematical model. Secondly, this model is solved using the finite element method. Error due to discretization of the mathematical model is introduced. The discretization error must be kept to a minimum. A coarse mesh density is not as flexible as the mathematical model with infinite degrees of freedom. Hence, the calculated natural frequencies will be overestimated. The model updating will result in physically incorrect material properties. Discretization error is estimated by comparing successive solutions with refined mesh density. More elements will result in a more exact solution of the mathematical model. When the difference between successive solutions is minimal, the mesh is "convergence". The accuracy of the analysis is related to the mesh density.

\subsection{Experimental error}

Incorrect input cannot result in physical correct material properties. Experimental error can be divided into two categories. Random errors can be treated with statistical procedures. Modal analysis software is capable in minimizing random errors. Systematic errors are a lot more difficult to detect and to solve. A damaged accelerometer will produce systematically an error on his output. Experimental tests on an analytical known problem can indicate a systematic error.

\subsection{Controllability}

A numerical model is controllable if it is possible to tune the model output from an arbitrary point $\{\mathbf{y}\}_{0}$ in the parameter space to a measured point $\{\mathbf{y}\}_{\exp }$ with the 
selected parameter set. Non-controllability can be turned into controllability by selecting more or more appropriate parameter. A parameter is appropriate if the sensitivity with respect to the response is sufficiently high.

\subsection{Observability}

A numerical model is observable if measurement of the output contains sufficient information for the identification of the selected parameters. A natural frequency does not contain information concerning the colour of the structure. It is impossible to identify a colour by measuring the natural frequency of the structure. Of course, one wants to identify all four orthotropic material properties by measuring a certain amount of frequencies. Unfortunately, depending on the structure, not every parameter will be identifiable.

To investigate observability, FEMtools offers sensitivity sum curves. Such a curve sums all sensitivity values for all responses as a function of parameter number. A low sensitivity sum value shows that none of the responses contains sufficient information for the identification of that parameter. If this occurs, one can add more experimental measurements or conclude that the parameter in question can not be identified by frequency measurement.

\subsection{Initial parameter values}

Model updating requires initial parameter values $\{\mathbf{p}\}_{0}$. The quality of the initial parameter values can affect both the speed of convergence and whether or not convergence to the "true" parameters is achieved. The parameter estimation problem is often posed as a problem of constraint minimization and in the case of non-linearity in parameters; a particular minimum is sought on a surface which contains many minima and maxima. Usually either a local minimum is sought, when there is confidence in the initial model, or else the problem is to determine the unique global minimum.

To obtain initial values for the longitudinal moduli $E_{x}$ and $E_{y}$, the first bending frequency of a beam specimen is determined. To identify $E_{x}$ a beam model in the span direction of the structure is used. To identify $E_{y}$ a beam model in the width direction of the structure is used. Knowledge of the bending frequency and dimensions of the specimen renders the elastic modulus of the material. The formula used to estimate the elasticity modulus [2]:

$$
\mathrm{E}=7.89 \mathrm{e}-2 *{\text { (frequency })^{2} *(\text { length })}^{4} * \text { density } * \text { transverse section } *
$$
(Moment of inertia) 


\section{Model updating: FEMtools}

\subsection{Sensitivity calculation}

The matrix [S] that appears in the linear Taylor term is called the sensitivity matrix. It contains the partial derivatives of the output components for the different parameter values. There are two basic approaches to compute sensitivities: (i) using differential sensitivities and (ii) using a finite difference approximation. Which one to use depends on the parameter type. For nonproportional parameters, such as the Young's modulus for orthotropic materials, FEMtools uses finite difference sensitivities. In this method derivatives are approximated with a forward finite difference approach. This is done using the results of two finite element analysis for two states of the parameter $\mathrm{p}_{\mathrm{j}}$. The element $i j$ of the matrix $[\mathbf{S}]$ becomes

$$
\frac{\Delta \mathrm{y}_{\mathrm{i}}}{\Delta \mathrm{p}_{\mathrm{j}}}=\frac{\mathrm{y}_{\mathrm{i}}\left(\mathrm{p}_{\mathrm{j}}-\Delta \mathrm{p}_{\mathrm{j}}\right)-\mathrm{y}_{\mathrm{i}}\left(\mathrm{p}_{\mathrm{j}}\right)}{\Delta \mathrm{p}_{\mathrm{j}}}
$$

The sensitivities discussed so far are absolute sensitivities. This means that they use the units of the response and parameter value. The absolute sensitivities can be made independent of the units used for the response and parameter values. They are then referred to as normalized sensitivities. A normalized sensitivity shows the percentage change of the response value for one percent change of the parameter value. The element $i j$ of the matrix $\left[\mathbf{S}_{n}\right]$ can be written as

$$
\mathrm{S}_{\mathrm{ij}(\mathrm{n})}=\frac{\Delta \mathrm{y}_{\mathrm{i}}}{\Delta \mathrm{p}_{\mathrm{j}}} \times \frac{\mathrm{p}_{\mathrm{j}}}{\mathrm{y}_{\mathrm{i}}}
$$

\subsection{Mode shape pairing}

During model updating, the algorithm will try to drive the predicted analytical response to the experimental reference data in the test database. This implies that the algorithm knows which analytical response has to match with which experimental response. This can be defined using sequential mode shape pairing. Sequential mode pairing means that analytical mode 1 will be paired with experimental mode 1, analytical mode 2 with experimental mode 2 , etc. If during model updating a switch of mode shapes occurs, this method fails. The resulting frequencies will probably be close but the mode shapes are different. There is no possibility to connect a mode shape to a particular experimental reference response.

To deal with the above problem, the program will copy the analytical mode shapes, predicted by the initial values for parameters $E_{x}, E_{y}, G_{\mathrm{xy}}$ and $v_{\mathrm{xy}}$, to the test database. The user of the program knows exactly the sequence of these modes [the program will previously show them]. It is now up to the user to connect the correct experimental reference frequency with the corresponding mode shape. In other words, the test database must reflect the correct physical 
response [natural frequency with corresponding mode shape]. Automatic mode shape pairing can now be used to drive the analytical response to the experimental reference response in the test database.

During model updating, automatic mode shape pairing makes a relation between those frequencies which have the highest Modal Assurance Criterion (MAC). The MAC is a measure of the squared cosine of the angle between two mode shapes. To compute the MAC between an analytical and experimental mode shape, the following equation is used:

$$
\operatorname{MAC}\left(\Psi_{\text {analyt }}, \Psi_{\text {exp }}\right)=\frac{\left|\left(\left\{\Psi_{a}\right\}^{T}\left\{\Psi_{e}\right\}\right)^{2}\right|}{\left(\left\{\Psi_{a}\right\}^{T}\left\{\Psi_{a}\right\}\right)\left(\left\{\Psi_{e}\right\}^{T}\left\{\Psi_{e}\right\}\right)}
$$

After model updating, a MAC value can be calculated between the updated fem model and the physical test modal. If no mode switch occurred during model updating this MAC matrix is a diagonal matrix.

\subsection{Aspects of convergence}

CCABSOLUTE: Average value of weighted absolute relative differences between predicted and reference resonance frequencies.

CCMEAN: Average value of weighted relative differences between predicted and reference resonance frequencies.

In model updating, the above correlation coefficients are interpreted as an objective function that needs to be minimized. With each iteration loop the values of the correlation coefficients will be verified to check if a convergence criterion is satisfied. The following criteria are used:

(1) The value of the reference correlation coefficient is less than an imposed margin:

$$
\mathrm{CC}_{\mathrm{t}}<\varepsilon_{1}
$$

(2) Two consecutive values of the reference correlation coefficient are within a given margin.

$$
\left|C C_{t+1}-C C_{t}\right|<\varepsilon_{2}
$$

The iteration loop in model updating will be stopped as soon as one of these tests is satisfied. Default values for $\varepsilon_{1}$ and $\varepsilon_{2}$ are used namely 0.08 .

\section{Example: symmetric U-profile}

\subsection{Section properties}

Dimensions according to figure 3 :

Moment of inertia $I_{y}: 439635 \mathrm{~mm}^{4}$

Moment of inertia $I_{z}: 251905 \mathrm{~mm}^{4}$

Torsional stiffness factor $J: 1990 \mathrm{~mm}^{4}$ 


\subsection{Volume properties}

Volume: $867793 \mathrm{~mm}^{3}$

Mass: 1599 gram

Density: $1.8426 \mathrm{e}-9 \mathrm{Mg} / \mathrm{mm}^{3}$

Length: $1470 \mathrm{~mm}$

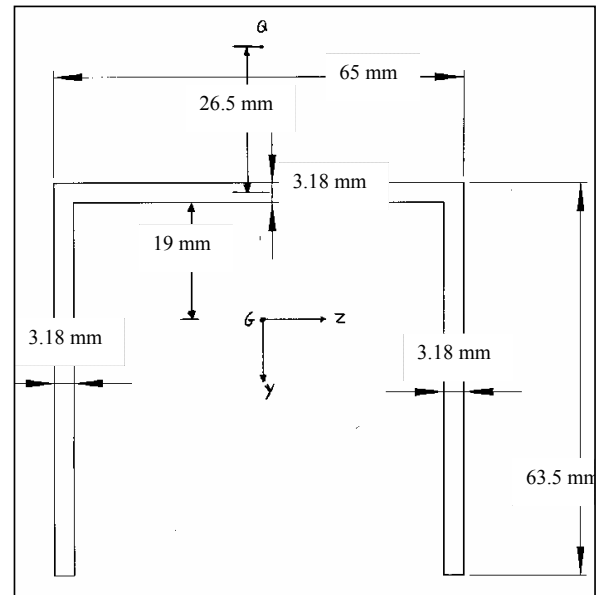

Figure 3: $\quad$ Symmetric U-profile.

\subsection{Estimation initial values}

An initial value for $E_{x}$ is obtained by measuring the first bending mode of a beam specimen in the span direction of the structure. An initial value for $E_{y}$ is obtained by measuring the first bending mode of a beam specimen in the width direction of the structure. Typical engineering properties of a glass-polymer composite are used for $G_{x y}$ and $v_{x y}[3]$.

\begin{tabular}{|c|c|c|}
\hline & $\begin{array}{c}\text { Initial } \\
\text { value } \\
\left(\mathbf{N} / \mathbf{m m}^{2}\right)\end{array}$ & $\begin{array}{c}\text { Method used } \\
\text { to obtain value }\end{array}$ \\
\hline $\mathbf{E x}$ & 22000 & Measured \\
\hline $\mathbf{E y}$ & 11800 & Measured \\
\hline $\mathbf{G x y}$ & 5000 & Literature \\
\hline $\mathbf{v x y}$ & 0,3 & Literature \\
\hline
\end{tabular}


234 High Performance Structures and Materials III

\subsection{Calculated natural frequencies and mode shapes (based on initial values)}
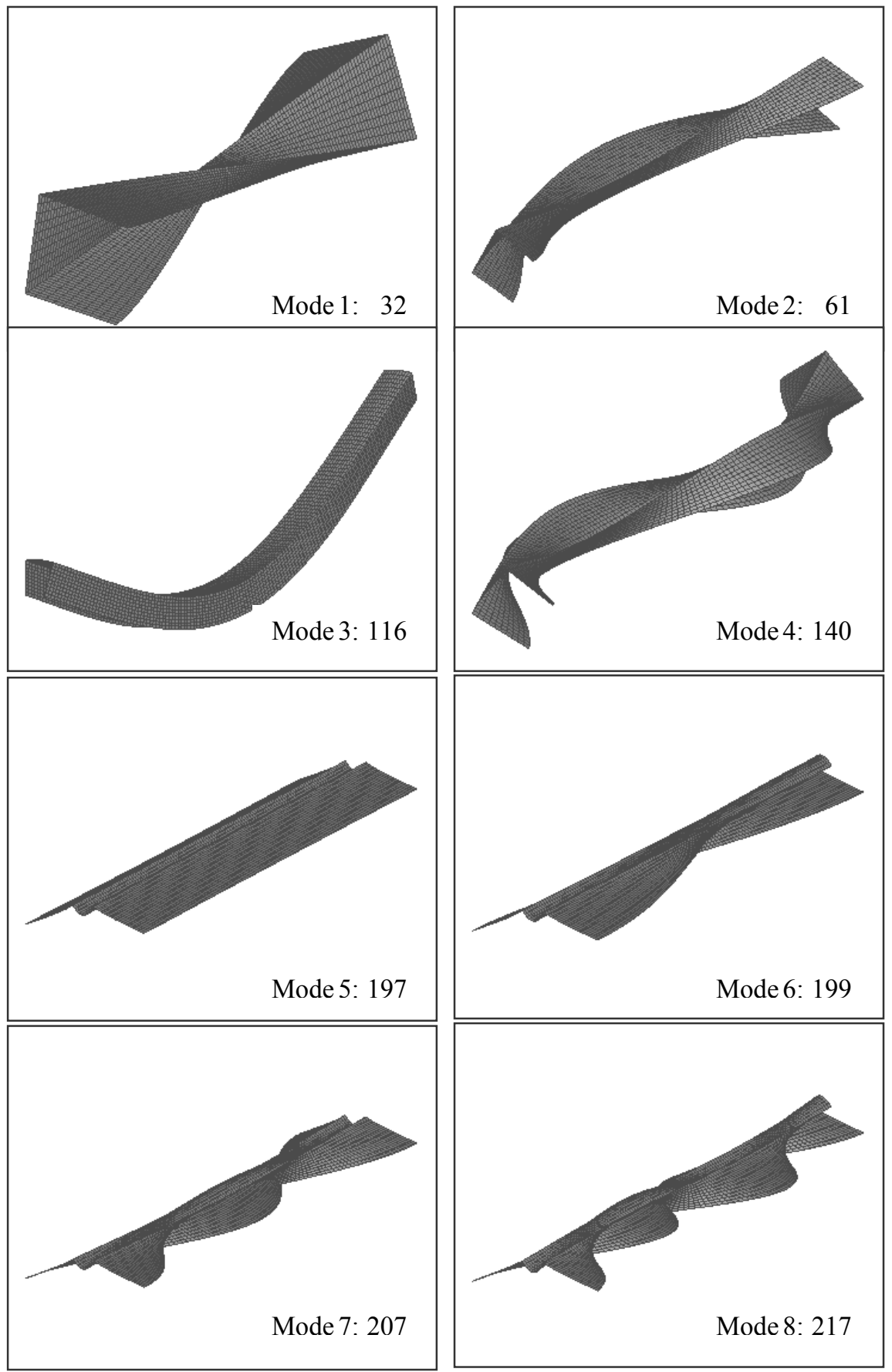

WIT Transactions on The Built Environment, Vol 85, (C) 2006 WIT Press www.witpress.com, ISSN 1743-3509 (on-line) 

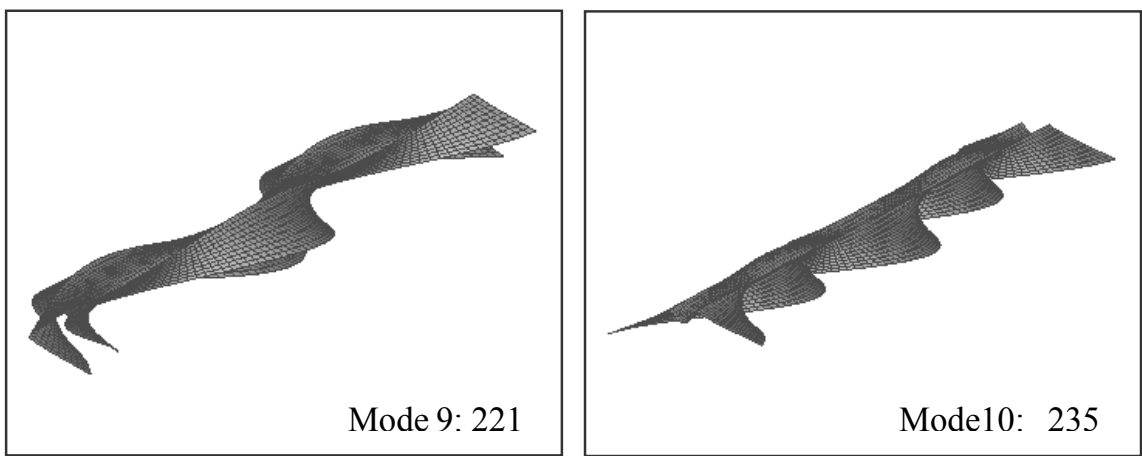

\subsection{Sensitivity matrix}

The sensitivity matrix shows the sensitivities of the four material parameters versus the first ten resonant frequencies. Parameter 1, 2, 3 and 4 equals respectively $E_{x}, E_{y}, G_{x y}$ and $v_{x y}$. Figure 4 shows the sensitivity matrix.

The sensitivity matrix indicates that the first torsion mode contains information about the shear modulus $G_{x y}$. The second mode - a complex bending mode around the $\mathrm{Y}$-axis - is sensitive to a change in value of $E_{x}$ and $G_{x y}$. The parameter $E_{y}$ can be identified by using resonant frequency five and six. None of the responses are sensitive to a change in value of $v_{x y}$. No attempt should be made to identify $v_{x y}$ by measuring natural frequencies.

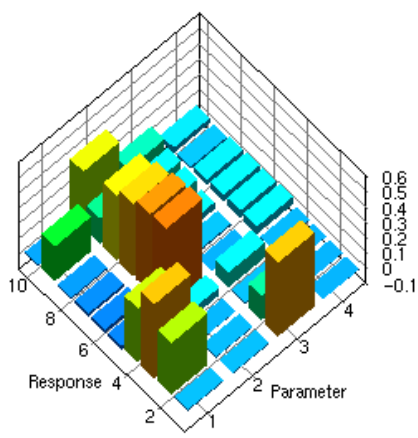

Figure 4: $\quad$ Sensitivity matrix.

\subsection{Model updating results}

Natural frequencies and corresponding mode shapes are measured using a laser vibrometer as measuring device and a shaker as excitation device. There was no indication, what so ever, of modes five and six in the experimental measured data. Probably due to the fact that a single shaker is not optimal to excite mode five. Additional, both modes are extremely close to each other. Consequently, further discussion will focus on the determination of $E_{x}$ and $G_{x y}$. The first four modes are used to identify $E_{x}$ and $G_{x y}$. 


\section{Conclusions}

This paper presents a method to determine the global effective orthotropic material properties by measuring a certain amount of natural frequencies of a composite beam structure. The program - developed in FEMtools - is used to determine the properties of composite beams with closed cross-sections and open cross-sections. Some general trends are clearly observed and stated hereafter.

Closed box beams behave relatively straightforward. In general, the first modes are bending modes around the principal axes of the cross-section. The torsion mode is found in a higher region since the torsional stiffness factor of a closed cross-section is rather high. The bending modes can be used to identify $E_{x}$ and the torsion mode can be used to identify $G_{x y}$. The natural frequencies of this kind of beams are not sensitive to a change in value of $E_{y}$ and $v_{x y}$. It is not possible to determine these parameters by measuring natural frequencies.

Beams with open cross-section are much more complex in behaviour and general conclusions can not be drawn. For this kind of beams, certain complex mode shapes are sensitive to a change in value of multiple parameters. Moreover, frequencies exists which are particularly sensitive to a modification of $E_{y}$ and can be used to identify this parameter. It is not possible to identify values for $v_{x y}$.

\begin{tabular}{|c|c|c|c|c|c|c|c|c|c|c|c|c|c|c|c|c|}
\hline & $\begin{array}{l}\text { iitial v } \\
{[\mathrm{N} / \mathrm{m}}\end{array}$ & $\begin{array}{l}\text { alues } \\
\left.\mathrm{m}^{2}\right]\end{array}$ & & & $\begin{array}{l}\text { Parar } \\
\text { selec }\end{array}$ & Imet & & $\begin{array}{r}\text { Fen } \\
\text { vers }\end{array}$ & $\begin{array}{l}\text { n data } \\
\text { initial } \\
\text { us Ex] }\end{array}$ & $\begin{array}{l}\text { based on } \\
\text { values } \\
\text { p. data }[\mathrm{Hz}]\end{array}$ & & $\begin{array}{l}\text { lode } \\
\text { lape } \\
\text { itch } \\
\text { ity }\end{array}$ & & $\begin{array}{l}\text { al para } \\
\text { lues [N] }\end{array}$ & $\begin{array}{l}\text { imeter } \\
\left./ \mathbf{m m}^{2}\right]\end{array}$ & \\
\hline Ex & Ey & Gxy & Vxy & Ex & Ey $\mathbf{C}$ & Gxy & Vxy & $\begin{array}{c}\text { Fem } \\
\text { value }\end{array}$ & $\begin{array}{l}\text { Exp. } \\
\text { value }\end{array}$ & $\begin{array}{c}\text { Description } \\
\text { mode }\end{array}$ & $\mathbf{Y}$ & $\mathbf{N}$ & Ex & Ey & Gxy & Vxy \\
\hline 22000 & 11800 & 5000 & 0,3 & $\mathrm{X}$ & & $\mathrm{X}$ & & 32 & 32.5 & 1 torsion & & $\mathrm{X}$ & 28944 & 11800 & 5014 & 0,3 \\
\hline & & & & & & & & 61 & 66.25 & $\begin{array}{l}1 \text { complex } \\
\text { bending Y }\end{array}$ & & & & & & \\
\hline & & & & & & & & 116 & 137.5 & 1 bending $Z$ & & & & & & \\
\hline & & & & & & & & 140 & 146.9 & $\begin{array}{l}2 \text { complex } \\
\text { bending Y }\end{array}$ & & & & & & \\
\hline
\end{tabular}

The program needs initial values for orthotropic material properties, before identification can start. A deviation of $25 \%$ given on estimated initial values, results in the same final updated values for the parameters. Hence, the final updated results are almost not sensitive to a deviation of initial values.

The program can also be used to study the influence of the length of the structure on the possibility to identify certain parameters. Consequently, an optimal length can be determined for which the first (two) frequencies are very sensitive to a change in value of a preferred parameter. Making it possible to identify orthotropic material properties in a more easy and structured manner. 
High Performance Structures and Materials III 237

\section{References}

[1] Modal Testing: Theory and Practice, DJ Ewins, Research studies press

[2] Engineering Vibration, DJ Inman, Prentice Hall

[3] Stress analysis of fiber-reinforced composite materials, MW Hyer, McGrawHill 